\title{
THE CONVERSE OF SCHUR'S LEMMA IN GROUP RINGS
}

\author{
M. Alaoui and A. Haily
}

Abstract

In this paper, we study the structure of group rings by means of endomorphism rings of their modules. The main tools used here, are the subrings fixed by automorphisms and the converse of Schur's lemma. Some results are obtained on fixed subrings and on primary decomposition of group rings.

\section{Introduction and Notations}

Let $M$ be a module over a ring $R$. If $M$ is simple, then Schur's lemma states that $\operatorname{End}_{R}(M)$ is a division ring (a skew field). However, the converse of this result does not hold in general, even when $R$ is artinian.

Definition 1. We shall say that a ring $R$ has the CSL property (abbreviation of: Converse of Schur's Lemma), or that $R$ is a CSL-ring, if every module is simple whenever its endomorphism ring is a division ring.

In [6], we have shown that a perfect ring $R$ is a CSL-ring if and only if $R$ is primary decomposable in the sense of [4]. In this note, we shall give some characterizations of perfect and primary decomposable group rings. To this aim, we shall use the results of [6] and [1] concerned by the converse of Schur's lemma in perfect rings. We start this work with the following remark:

Let $A$ be a ring, $A[G]$ the group ring of a finite group $G$ over $A$. If $M$ is an $A[G]$-module, then $M$ is an $A$-module and for every $g \in G$, the mapping $L_{g}: M \rightarrow M$ defined by $L_{g}(x)=g x$, is an automorphism of the $A$-module $M$. Let $L: G \rightarrow \operatorname{Aut}_{A}\left(\operatorname{End}_{A}(M)\right)$ such that $g \mapsto L_{g}$ where

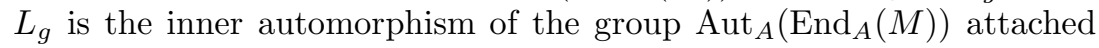

2000 Mathematics Subject Classification. 16S50, 16S34.

Key words. Modules, group ring, Schur's lemma.

This work has been partially supported by El Ministerio de Ciencia y Tecnología, proyecto BFM 2001-2335. Spain. 
to $L_{g}: L_{g}(u)=L_{g} u L_{g^{-1}}$ for all $u \in \operatorname{End}_{A}(M)$. For each $u \in \operatorname{End}_{A}(M)$ we have:

$$
\begin{aligned}
u \in \operatorname{End}_{A[G]}(M) & \Leftrightarrow u \in \operatorname{End}_{A}(M) \text { and } u(g x)=g u(x), \quad \forall g \in G, \forall x \in M \\
& \Leftrightarrow u \in \operatorname{End}_{A}(M) \text { and } g^{-1} u g=u, \quad \forall g \in G \\
& \Leftrightarrow u \in \operatorname{End}_{A}(M) \text { and } L_{g} u L_{g^{-1}}=u, \quad \forall g \in G .
\end{aligned}
$$

Consequently, $\operatorname{End}_{A[G]}(M)=\operatorname{End}_{A}(M)^{G^{\prime}}$ where $G^{\prime}=\left\{L_{g} \mid g \in G\right\}$, hence the results on fixed subrings can be used to study the properties of $\operatorname{End}_{A[G]}(M)$ and it will provide some information on the structure of $A[G]$.

The work is divided in two parts. In the first, we consider some ringtheoretical properties which remain true when passing from the fixed subring $R^{G}$ to the whole ring $R$. In this context, we study when regular elements of $R^{G}$ remain regular in $R$ (Corollary 5 ). The results are obtained by imposing some condition on $G$ and on $R$.

In the second part, we apply these results to study the $\operatorname{End}_{A[G]}(M)$, the endomorphism ring of $M$. This enables us to derive condition on $G$ that imply the primary decomposability of $A[G]$. We therefore pursue the study we made in [1].

(For the terminology and notations used here we refer to [2], [4].)

All rings considered in this work are associative with identity, and all the modules are left unitary modules. If $M$ is a module over a ring $R$, the endomorphism ring of $M$ is denoted by $\operatorname{End}_{R}(M)$.

- A ring $R$ is said to be perfect if it is left and right perfect.

- A ring $R$ is said to be primary, if the factor ring $R / J(R)$, where $J(R)$ denotes the Jacobson radical of $R$, is simple Artinian. Any primary left or right perfect ring is isomorphic to a full matrix ring over a local ring $[4]$.

- A right or left perfect ring $R$ is said to be primary decomposable, if it is isomorphic to a (finite) product of primary rings.

- It can be shown that, for a perfect and CSL-ring $R$, each factor ring of $R$ is primary decomposable. This is true because a CSL-property is conserved by passing to factors.

- If $M$ is an abelian group, $G$ a group of automorphisms of $M$, we write $M^{G}=\{x \in M \mid \sigma(x)=x, \forall \sigma \in G\}$ for the set of elements of $M$ fixed by $G$. This is clearly a subgroup of $M$. If $L$ is a subgroup of $M$ 
stable by $G$ ( $G$-stable), we note also $L^{G}=\{x \in L \mid \sigma(x)=x, \forall \sigma \in G\}$. Notice that if $G=H P$, where $H$ and $P$ are two subgroups of $G$ such that $H$ is normal in $G$, then $M^{G}=\left(M^{H}\right)^{P}$.

- For every $n \in \mathbb{N}^{*}$, we note $T_{n}(M)=\{x \in M \mid n x=0\}$ the $n$-torsion subgroup of $M$. This is a fully invariant subgroup of $M$, and if $T_{n}(M)=0$, we say that $M$ is $n$-torsion-free. $M$ is said to be torsion free, if $M$ is $n$-torsion-free for every $n \in \mathbb{N}^{*}$.

\section{CSL-property in group rings}

Lemma 2. Let $M$ be an abelian group, $p$ a prime integer and $G$ a finite p-group of automorphisms of $M$. If $M^{G}=0$, then $M$ is p-torsion free.

Proof: Assume that $L=T_{p}(M) \neq 0$. Then $L$ is a $G$-stable $p$-torsion group, and it can be viewed as a $\mathbb{F}_{p}$-vector space, where $\mathbb{F}_{p}$ is the finite field of $p$ elements and $G$ a group of automorphisms of the $\mathbb{F}_{p}$-space $L$. We are going to show that $L^{G} \neq(0)$.

- Suppose first that $G=\langle\sigma\rangle$ is cyclic of order $p^{k}$, then

$$
\left(\sigma-\mathrm{id}_{M}\right)^{p^{k}}=\sigma^{p^{k}}-\mathrm{id}_{M}=0
$$

so $\sigma-\operatorname{id}_{M}$ is nilpotent and hence $\operatorname{ker}\left(\sigma-\mathrm{id}_{M}\right) \neq 0$. This implies that $L^{G} \neq 0$.

- We now argue by induction on $|G|$ the order of $G$. If $|G|=p$, then $G$ is a cyclic $p$-group; we apply the last situation.

Let us assume that the lemma holds for $p$-groups of order $<p^{k}$ where $k>1$. If $G$ is a $p$-group of order $p^{k}$, then by elementary group theory, $G$ contains a normal subgroup $H$ of order $p^{k-1}$. Hence $G / H$ is cyclic. Put $G / N=g r\langle\bar{\sigma}\rangle$. We have $G=N \cup N \sigma \cup N \sigma^{2} \cup \cdots \cup N \sigma^{p-1}$ so $G=N . H$ where $H=g r\langle\sigma\rangle$, the subgroup generated by $\sigma$. Now using the induction hypothesis, one obtains $L^{N} \neq 0$. Thus, since $H$ is cyclic, the last case show that $\left(L^{N}\right)^{H} \neq(0)$ so $L^{G}=\left(L^{N)^{H}} \neq(0)\right.$.

We will need the following version of Bergman-Isaacs's theorem:

Theorem 3 ([3] or $[\mathbf{1 4}$, Corollary 2.5.53]). Let $R$ be a ring not necessarily unitary and $G$ a finite group of automorphisms of $R$. If $R$ is $|G|$-torsion free and $R$ is not nilpotent, then $R^{G} \neq 0$.

Recall that for a finite group $G$ and a prime integer $p$ we say that $g \in G$ is a $p^{\prime}$-element, if the order of $g$ is prime to $p$. Moreover, if the 
set $H$ of $p^{\prime}$-elements is a subgroup of $G$, then $H$ is normal in $G$ and $G=H P$, where $P$ is a $p$-Sylow subgroup of $G$.

Theorem 4. Let $R$ be a semiprime ring, $G$ a finite group of automorphisms of $R$. Assume that for every prime integer $p$ for which $T_{p}(R) \neq 0$, the set of $p^{\prime}$-elements of $G$ is a subgroup of $G$. Then for every $G$-stable nonzero left or right ideal $I$ we have $I^{G} \neq 0$.

Proof: • Let $I$ be a nonzero $G$-stable left (resp. right) ideal. If $I$ is torsion free, since $R$ is semi prime then the Bergman-Isaacs theorem (see [3] or Theorem 3 or $\left[\mathbf{1 4}\right.$, Theorem 2.5.52, p. 198]), says $I^{G} \neq 0$.

- If $I$ is not torsion free, then $T(I) \neq 0$ and so $A=T_{p}(I) \neq 0$ for some prime number $p$ dividing $|G|$. Thus, $A$ is a nonzero $p$-torsion left (resp. right ideal) of $R$. Now, since $T_{p}(I) \subseteq T_{p}(R)$, by hypothesis, we have $G=H P$ where $H$ is a normal $p^{\prime}$-subgroup of $G$ and $P$ is a Sylow $p$-subgroup. Thus, $A^{G}=\left(I^{H}\right)^{P}$. Since $R$ is semiprime, then $A$ is not nilpotent and the Bergman-Isaacs theorem implies $L=A^{H} \neq 0$. Now Lemma 2 implies $A^{G}=L^{P} \neq 0$ as required.

The same argument is valid if we change the word "left" by "right".

Recall that a ring $R$ is said to be a quotient ring, if every regular element of $R$ is invertible. Quotient rings are called classical rings in [9].

Corollary 5. Let $R$ be a semiprime ring, $G$ a finite group of automorphisms of $R$. Assume that for every prime integer $p$ such that $R$ has $p$-torsion elements, the set of $p^{\prime}$-element of $G$ is a subgroup of $G$. Then:

(i) Every left (resp. right) regular element in $R^{G}$ is left (resp. right regular) in $R$.

(ii) If $R$ is a quotient ring then so is $R^{G}$.

Proof: (i) Let $a \in R^{G}$ left regular in $R^{G}$ suppose that $a$ is not left regular in $R$ then, $I=\operatorname{Ann}_{d}(a)=\{b \in R: a b=0\}$ is right ideal, $G$-invariant since $a b=0 \Rightarrow a \sigma(b)=\sigma(a b)=0, \forall \sigma \in G$. Since $R$ is semiprime, by Theorem $3, I^{G} \neq 0$. Hence there exists a nonzero $b \in R^{G}$ such that $a b=0$, contradiction.

(ii) If $a \in R^{G}$ is left and right regular in $R^{G}$, then by (i), $a$ is left and right regular in $R$. Since $R$ is a quotient ring, $a$ is invertible in $R$. Thus, there is $c \in R$ such that $a c=c a=1$. For every $\sigma \in G, a \sigma(c)=\sigma(c) a=1$. Thus $\sigma(c)=c$ for all $\sigma \in G$ and hence $a$ is invertible in $R^{G}$.

We can now prove the main theorem: 
Theorem 6. Let $A$ a commutative and perfect ring, $G$ a finite group. The following assertions are equivalents:

(i) $A[G]$ is a primary decomposable ring.

(ii) $A[G]$ is CSL-ring.

(iii) For each prime number $p$ such that $T_{p}(A) \neq 0$, there exists a $p^{\prime}$-subgroup $H$ of $G$, and a $p$-Sylow subgroup $P$ of $G$ such that: $G=H P$.

Proof: - Since $G$ is finite group and $A$ is perfect ring, then the group ring $A[G]$ is perfect (cf. [13] or [15]). The equivalence of (i) and (ii) is a consequence of $[\mathbf{6}$, Theorem 3$]$.

- (i) $\Rightarrow$ (iii) Let $p$ a prime number such that $T_{p}(A) \neq 0$. We have $p A$ is proper ideal of $A$. By Zorn's lemma, $p A$ is contained in a maximal ideal $I$. Hence the factor $\operatorname{ring} A / I=K$ is a field (commutative) of characteristic $p$. Since $A[G]$ is a primary decomposable ring, so is $K[G]$ (see the Introduction). Now, we can apply the results of $[\mathbf{1}]$ : there exists a $p^{\prime}$-subgroup $H$ of $G$, and a $p$-Sylow subgroup $P$ of $G$ such that: $G=H P$

- (iii) $\Rightarrow$ (ii) Let $M$ a $A[G]$-module such that $D=\operatorname{End}_{A[G]}(M)$ is a division ring. We are going to show that $M$ is a simple $A[G]$-module.

First, put $I=\operatorname{Ann}_{A}(M)$, the annihilator of $M$ in $A$ and show, that $I$ is a maximal ideal of $A$. Since $A$ is commutative, then for each $a \in A$, the map $\rho_{a}: M \rightarrow M, m \mapsto \rho_{a}(m)=a m$ is an endomorphism of the $A[G]$-module $M$ and so the map $\rho A \rightarrow \operatorname{End}_{A[G]}(M), a \mapsto \rho(a)=\rho_{a}$ is a homomorphism of rings. It is clear that $I$ is the kernel of $\rho$. It follow that the factor ring $A / I$ is isomorph to a subring of a division ring $D$. Hence $A / I$ is a domain so the ideal $I$ is prime. But $A$ is perfect by hypothesis; so $I$ is maximal and $K=A / I$ is a commutative field.

Now, let $N$ be a nonzero submodule of the $A[G]$-module $M$, and consider the set $\mathcal{I}=\left\{u \in \operatorname{End}_{K}(M) \mid u(N)=0\right\}$. Then $\mathcal{I} \neq 0$. However, for each $u \in \mathcal{I}, g \in G$ and $x \in N$ we have: $L_{g} u L_{g^{-1}}(x)=g u\left(g^{-1} x\right)=0$ since $N$ is a $A[G]$-submodule of $M$. This show that $\mathcal{I}$ is a nonzero left ideal $G^{\prime}$-invariant of $\operatorname{End}_{A}(M)$ where $G^{\prime}=\left\{L_{g} \mid g \in G\right\}$.

Let $R=\operatorname{End}_{K}(M)=\operatorname{End}_{A}(M)$ (because $K=A / I$ and $I=\operatorname{Ann}_{A}(M)$ ). Then $R$ is a von Neumann regular ring and hence semiprime. But $G^{\prime}=$ $L(G)$ is a group of automorphisms of $R$, homomorphic image of $G$; by Theorem 3 we have $\mathcal{I}^{G^{\prime}} \neq 0$. Thus, it exists a nonzero $u$ lie in $R$ such that $u$ is $G^{\prime}$-invariant: $u \in \operatorname{End}_{K[G]}(M)$. Since $\operatorname{End}_{A[G]}(M)$ is a division ring, so it is for $\operatorname{End}_{K[G]}(M)$. Finally, $N=0$ because $u(N)=0$. 
As application, we can give a new characterization of nilpotent finite groups using a primary decomposition of the group ring $(\mathbb{Z} / n \mathbb{Z})[G]$, $n \in \mathbb{N}^{*}$ :

Corollary 7. Let $G$ be a finite group of order $n$. Then, $(\mathbb{Z} / n \mathbb{Z})[G]$ is primary decomposable, if and only if, $G$ is nilpotent group.

Acknowlegement. The authors wish to thank the referee for her/his helpful suggestions.

\section{References}

[1] M. Alaoui And A. Haily, The converse of Schur's Lemma in Noetherian rings and group algebras, Comm. Algebra 33(7) (2005), 2109-2114.

[2] F. W. Anderson And K. R. Fuller, "Rings and categories of modules", Second edition, Graduate Texts in Mathematics 13, Springer-Verlag, New York, 1992.

[3] G. M. Bergman and I. M. IsaAcs, Ring with fixed-point-free group actions, Proc. London Math. Soc. (3) 27 (1973), 69-87.

[4] C. FAith "Algebra. II. Ring Theory", Grundlehren der Mathematischen Wissenschften 191, Springer-Verlag, Berlin-New York, 1976.

[5] C. FAITH, Indecomposable injective modules and a theorem of Kaplansky, Comm. Algebra 30(12) (2002), 5875-5889.

[6] A. Haily and M. Alaoui, Perfect rings for which the converse of Schur's lemma holds, Publ. Mat. 45(1) (2001), 219-222.

[7] A. HAILY AND A. KAIDI, Caractérisation de certaines classes d'anneaux par des propriétés des endomorphismes de leurs modules, Comm. Algebra 27(10) (1999), 4943-4951.

[8] Y. Hirano And J. K. PARK, Rings for which the converse of Schur's lemma holds, Math. J. Okayama Univ. 33 (1991), 121-131.

[9] T. Y. LAM, "Lectures on modules and rings", Graduate Texts in Mathematics 189, Springer-Verlag, New York, 1999.

[10] J. C. McConnell And J. C. Robson, "Noncommutative Noetherian rings", with the cooperation of L. W. Small, Pure and Applied Mathematics (New York), A Wiley-Interscience Publication, John Wiley \& Sons, Ltd., Chichester, 1987.

[11] R. S. PIERCE, "Associative algebras", Graduate Texts in Mathematics 88, Studies in the History of Modern Science 9, SpringerVerlag, New York-Berlin, 1982.

[12] G. Renault, "Algèbre non commutative", Collection "Varia Mathematica", Gauthier-Villars Éditeur, Paris-Brussels-Montreal, Que., 1975 . 
[13] G. Renault, Sur les anneaux de groupes, C. R. Acad. Sci. Paris Sér. A-B 273 (1971), A84-A87.

[14] L. H. Rowen, "Ring theory", Student edition, Academic Press, Inc., Boston, MA, 1991.

[15] S. M. Woods, On perfect groups rings, Proc. Amer. Math. Soc. 27 (1971), 49-52.

Département de Mathématiques et Informatique

Faculté des Sciences

BP 20 El Jadida

Morocco

E-mail address: alaoui@Math.net

E-mail address: haily@Math.net

Primera versió rebuda el 30 de maig de 2005, darrera versió rebuda el 27 de setembre de 2005. 